Supporting information for

\title{
Synthesis, Herbicidal Activity and QSAR of Novel $N$-Benzothiazolyl- pyrimidine-2,4-diones as Protoporphyrinogen Oxidase Inhibitors
}

Zuo Yang, ${ }^{\dagger}$ Wu Qiongyou, ${ }^{\dagger}$ Su Sun-wen, ${ }^{\dagger}$ Niu Cong-wei ${ }^{\ddagger}$ Xi Zhen ${ }^{\ddagger}$ and Yang Guang-Fu ${ }^{\dagger,+*}$

${ }^{\dagger}$ Key Laboratory of Pesticide \& Chemical Biology of Ministry of Education, College of Chemistry, Central China Normal University, Wuhan 430079, P.R.China;

ॠState Key Laboratory of Elemento-Organic Chemistry, Nankai University, Tianjin 300071, P. R. China

\section{Corresponding author:}

Prof. Guang-Fu Yang

College of Chemistry, Central China Normal University

Key Laboratory of Pesticide \& Chemical Biology, Ministry of Education

Wuhan 430079, P. R. China

Tel: +86-27-67867800

Fax: +86-27-67867141

E-mail: gfyang@mail.ccnu.edu.cn 


\section{Preparation of 2,4-disubtituted phenylcarbamates, 2a-c.}

To a stirred solution of 2,4-disubstituted aniline $(0.05 \mathrm{~mol})$ in $100 \mathrm{~mL}$ of anhydrous methylene chloride was added pyridine ( $7.9 \mathrm{~g}, 0.1 \mathrm{~mol})$ under ice bath, and followed by dropwise addition of a solution of methyl chloroacetate $(5.4 \mathrm{~g}, 0.05 \mathrm{~mol})$ in $20 \mathrm{~mL}$ of anhydrous methylene chloride within $30 \mathrm{~min}$. The reaction mixture was stirred for an additional $1 \mathrm{~h}$ and extracted with methylene chloride, and the combined extracts were washed with $2 \mathrm{~N}$ hydrochloric acid. The organic layer was dried over sodium sulfate, filtered, and concentrated under reduced pressure to give 2,4-disubtituted phenylcarbamates.

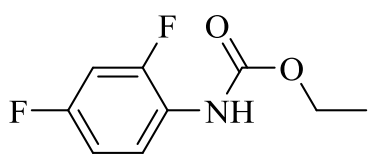

Data for 2a. Yield: $98 \%$, m.p. $48-49^{\circ} \mathrm{C} .{ }^{1} \mathrm{H}$ NMR $\left(600 \mathrm{MHz}, \mathrm{CDCl}_{3}\right) \delta 8.03(\mathrm{~s}, 1 \mathrm{H})$, $6.88-6.83(\mathrm{~m}, 2 \mathrm{H}), 6.69-6.66(\mathrm{~m}, 1 \mathrm{H}), 4.24(\mathrm{q}, J=7.2 \mathrm{~Hz}, 2 \mathrm{H}), 1.33(\mathrm{t}, J=7.2 \mathrm{~Hz}$, 3H). ESI-MS: 224.1(M+Na $)^{+}$.

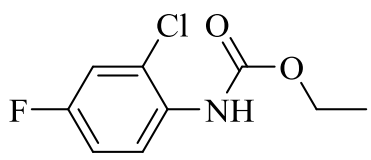

Data for 2b. Yield: 96\%, m.p. $42-43{ }^{\circ} \mathrm{C} .{ }^{1} \mathrm{H} \mathrm{NMR}\left(400 \mathrm{MHz}, \mathrm{CDCl}_{3}\right) \delta 8.12(\mathrm{~s}, 1 \mathrm{H})$, $7.13-7.10(\mathrm{~m}, 1 \mathrm{H}), 7.03-6.98(\mathrm{~m}, 2 \mathrm{H}), 4.24(\mathrm{q}, J=7.2 \mathrm{~Hz}, 2 \mathrm{H}), 1.33(\mathrm{t}, J=7.2 \mathrm{~Hz}$, 3H). ESI-MS: 240.1(M+Na $)^{+}$.

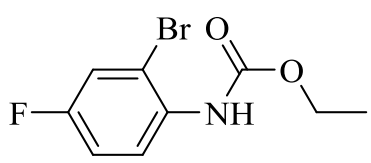


Data for 2c. Yield: 98\%, m.p. 53-55 C. ${ }^{1} \mathrm{H}$ NMR (600 MHz, $\left.\mathrm{CDCl}_{3}\right) \delta 8.10(\mathrm{~s}, 1 \mathrm{H})$, $7.36-7.23(\mathrm{~m}, 1 \mathrm{H}), 7.12-7.01(\mathrm{~m}, 1 \mathrm{H}), 6.97(\mathrm{~s}, 1 \mathrm{H}), 4.24(\mathrm{q}, J=7.2 \mathrm{~Hz}, 2 \mathrm{H}), 1.33(\mathrm{t}$, $J=7.2 \mathrm{~Hz}, 3 \mathrm{H}) . \mathrm{ESI}-\mathrm{MS}: 284.3(\mathrm{M}+\mathrm{Na})^{+}$.

\section{Preparation of ethyl 3-amino-4,4,4-trifluorobut-2-enoate, 3.}

To a stirred solution of ethyl 4,4,4-trifluoroacetoacetate (18.4 g, $0.1 \mathrm{~mol})$ in ethanol $(125 \mathrm{~mL})$ was added ammonium acetate $(30.8 \mathrm{~g}, 0.4 \mathrm{~mol})$ and the mixture was refluxed for $8 \mathrm{~h}$. After cooling to room temperature, the solution was poured into water and extracted with methyl chloroacetate. The organic layer was dried over sodium sulfate, filtered, and concentrated under reduced pressure to give ethyl 3-amino-4,4,4-trifluorobut-2-enoate<smiles>CCOC(=O)/C=C(\N)C(F)(F)F</smiles>

Data for 3. Yield: $88 \%$, White oil. ${ }^{1} \mathrm{H}$ NMR $\left(600 \mathrm{MHz}, \mathrm{CDCl}_{3}\right) \delta 6.22(\mathrm{~s}, 2 \mathrm{H}), 5.13$ $(\mathrm{s}, 1 \mathrm{H}), 4.18(\mathrm{q}, J=6.6 \mathrm{~Hz}, 2 \mathrm{H}), 1.29(\mathrm{t}, J=6.6 \mathrm{~Hz}, 3 \mathrm{H})$. ESI-MS: $184.1(\mathrm{M}+\mathrm{H})^{+}$. phenyl)-1-methyl-6-(trifluoromethyl)pyrimidine- 2,4-diones, 5a-c.

To a stirred solution of $\mathbf{3}(7.3 \mathrm{~g}, 0.04 \mathrm{~mol})$ in $100 \mathrm{~mL}$ anhydrous dimethylformamide was added a $60 \%$ sodium hydride $(1.92 \mathrm{~g}, 0.048 \mathrm{~mol})$ at $0{ }^{\circ} \mathrm{C}$ and the mixture was stirred for $30 \mathrm{~min}$. Thereafter, a solution of the carbamate, 2 , in $50 \mathrm{~mL}$ anhydrous dimethylformamide was added dropwise within $30 \mathrm{~min}$, the mixture was stirred for 30 
min and heated to $120^{\circ} \mathrm{C}$ for $6 \mathrm{~h}$ to synthesize the product, 4 . The reaction mixture was cooled to room temperature and potassium carbonate was added $(6.6 \mathrm{~g}, 0.048$ mol), then the reaction mixture was treated with methyl iodide $(11.4 \mathrm{~g}, 0.08 \mathrm{~mol})$ and stirred overnight. The reaction mixture was poured into $800 \mathrm{~mL}$ of ice water and the precipitate was collected by filtration, washed with water and dried to give the dione,

5.<smiles>Cn1c(C(F)(F)F)cc(=O)n(-c2ccc(F)cc2F)c1=O</smiles>

Data for 5a. Yield: 67\%, m.p. 89-90 ${ }^{\circ} \mathrm{C} .{ }^{1} \mathrm{H}$ NMR $\left(600 \mathrm{MHz}, \mathrm{CDCl}_{3}\right) \delta 7.80$ (q, $J=$ $4.8 \mathrm{~Hz}, 1 \mathrm{H}), 7.02-6.99(\mathrm{~m}, 1 \mathrm{H}), 7.43$ (t, $J=8.4 \mathrm{~Hz}, 1 \mathrm{H}), 6.37(\mathrm{~s}, 1 \mathrm{H}), 3.56(\mathrm{~s}, 3 \mathrm{H})$. ESI-MS: $329.1(\mathrm{M}+\mathrm{Na})^{+}$.<smiles>Cn1c(C(F)(F)F)cc(=O)n(-c2ccc(F)cc2Cl)c1=O</smiles>

Data for 5b. Yield: $63 \%$, m.p. $103-104^{\circ} \mathrm{C} .{ }^{1} \mathrm{H}$ NMR $\left(600 \mathrm{MHz}, \mathrm{CDCl}_{3}\right) \delta 7.31(\mathrm{q}, J$ $=3.0 \mathrm{~Hz}, 1 \mathrm{H}), 7.24(\mathrm{t}, J=3.6 \mathrm{~Hz}, 1 \mathrm{H}), 7.15-7.12(\mathrm{~m}, 1 \mathrm{H}), 6.38(\mathrm{~s}, 1 \mathrm{H}), 3.57(\mathrm{~s}, 3 \mathrm{H})$. ESI-MS: 323.1(M+H $)^{+}$.<smiles>Cn1c(C(F)(F)F)cc(=O)n(-c2ccc(F)cc2Br)c1=O</smiles> 
Data for 5c. Yield: $65 \%$, m.p. $115-116^{\circ} \mathrm{C} .{ }^{1} \mathrm{H}$ NMR $\left(600 \mathrm{MHz}, \mathrm{CDCl}_{3}\right) \delta 7.80(\mathrm{q}, J=$ $2.4 \mathrm{~Hz}, 1 \mathrm{H}), 7.59(\mathrm{~d}, J=8.4 \mathrm{~Hz}, 1 \mathrm{H}), 7.43(\mathrm{t}, J=8.4 \mathrm{~Hz}, 1 \mathrm{H}), 6.59(\mathrm{~s}, 1 \mathrm{H}), 3.41(\mathrm{~s}$, 3H). ESI-MS: $389.0(\mathrm{M}+\mathrm{Na})^{+}$.

Preparation

of

\section{3-(2',4'-disubstituted-5'-nitrophenyl)-1-methyl-6-(trifluoromethyl)-pyrimidine-2,}

4-dione, 6a-c.

To a stirred solution of $5(0.026 \mathrm{~mol})$ in $52 \mathrm{~mL}$ of concentrated $\mathrm{H}_{2} \mathrm{SO}_{4}$ was slowly added $68 \%$ nitric acid $(3.12 \mathrm{~g}, 0.031 \mathrm{~mol})$ at $0^{\circ} \mathrm{C}$. The temperature was maintained at $0^{\circ} \mathrm{C}$ for $1 \mathrm{~h}$, and then the reaction mixture was poured into ice water. The precipitate was collected by filtration, washed with water and dried to give 3-(2',4'-disubstituted-5'-nitrophenyl)-1-methyl-6-(trifluoro-methyl)pyrimidine-2,4-di one.<smiles>Cn1c(C(F)(F)F)cc(=O)n(-c2cc([N+](=O)[O-])c(F)cc2F)c1=O</smiles>

Data for 6a. Yield: $95 \%$, m.p. $147-149^{\circ} \mathrm{C} .{ }^{1} \mathrm{H}$ NMR $\left(600 \mathrm{MHz}, \mathrm{CDCl}_{3}\right) \delta 8.16(\mathrm{t}, J=$ $7.2 \mathrm{~Hz}, 1 \mathrm{H}), 8.30(\mathrm{~d}, J=9.6 \mathrm{~Hz}, 1 \mathrm{H}), 6.40(\mathrm{~s}, 1 \mathrm{H}), 3.58(\mathrm{~s}, 3 \mathrm{H})$. ESI-MS: 374.1(M+Na) .<smiles>Cn1c(C(F)(F)F)cc(=O)n(-c2cc([N+](=O)[O-])c(F)cc2Cl)c1=O</smiles> 
Data for 6b. Yield: $97 \%$, m.p. $131-132{ }^{\circ} \mathrm{C} .{ }^{1} \mathrm{H}$ NMR $\left(600 \mathrm{MHz}, \mathrm{CDCl}_{3}\right) \delta 8.10(\mathrm{~d}, J$ $=7.2 \mathrm{~Hz}, 1 \mathrm{H}), 7.56(\mathrm{~d}, J=9.6 \mathrm{~Hz}, 1 \mathrm{H}), 6.41(\mathrm{~s}, 1 \mathrm{H}), 3.59(\mathrm{~s}, 3 \mathrm{H})$. ESI-MS: $390.0(\mathrm{M}+\mathrm{Na})^{+}$.

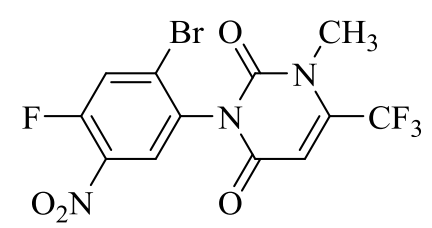

Data for 6c. Yield: $97 \%$, m.p. $127-129^{\circ} \mathrm{C} .{ }^{1} \mathrm{H}$ NMR $\left(600 \mathrm{MHz}, \mathrm{CDCl}_{3}\right) \delta 8.49(\mathrm{~d}, J=$ $6.6 \mathrm{~Hz}, 1 \mathrm{H}), 8.30(\mathrm{~d}, J=10.8 \mathrm{~Hz}, 1 \mathrm{H}), 6.69(\mathrm{~s}, 1 \mathrm{H}), 3.50(\mathrm{~s}, 3 \mathrm{H})$. ESI-MS: 434.1( $(\mathrm{M}+\mathrm{Na})^{+}$.

Preparation of

\section{3-(5'-amino-2',4'-disubstitutedphenyl)-1-methyl-6-(trifluoromethyl)-pyrimidine-}

\section{2,4-dione, 7a-c.}

Iron powder $(2.67 \mathrm{~g}, 0.05 \mathrm{~mol})$ was added portionwise to a stirred solution of $\mathbf{6}(0.025$ $\mathrm{mol}), \mathrm{NH}_{4} \mathrm{Cl}(2.66 \mathrm{~g}, 0.05 \mathrm{~mol})$ in a mixture of ethanol $(75 \mathrm{~mL})$ and water $(10 \mathrm{~mL})$ at reflux temperature. The reaction mixture was refluxed for $6 \mathrm{~h}$ and filtered through diatomaceous earth, and the filtrate was concentrated under reduced pressure. The residue was extracted with ethyl acetate, and the organic residue was washed with brine. Then the residue was dried over sodium sulfate, filtered, and concentrated under reduced pressure to give 3-(5'-amino-2',4'-disubstitutedphenyl)-1-methyl-6-(trifluoromethyl)pyrimidine-2,4-di one. 


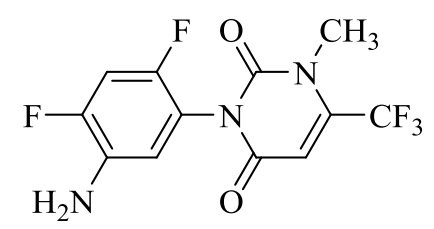

Data for 7a. Yield: $86 \%$, m.p. $166-167^{\circ} \mathrm{C} .{ }^{1} \mathrm{H}$ NMR (600 MHz, DMSO-d6) $\delta 7.24(\mathrm{t}$, $J=10.8 \mathrm{~Hz}, 1 \mathrm{H}), 6.73(\mathrm{t}, J=8.4 \mathrm{~Hz}, 1 \mathrm{H}), 6.52(\mathrm{~s}, 1 \mathrm{H}), 5.23(\mathrm{~s}, 2 \mathrm{H}), 3.40(\mathrm{~s}, 3 \mathrm{H})$. ESI-MS: 284.3(M+Na) ${ }^{+}$ESI-MS: $322.1(\mathrm{M}+\mathrm{H})^{+}$.

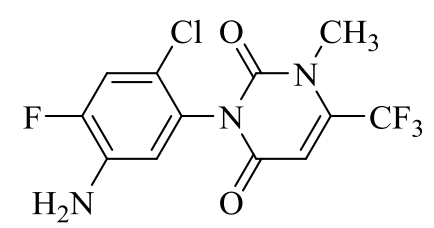

Data for 7b. Yield: $89 \%$, m.p. $157-159^{\circ} \mathrm{C} .{ }^{1} \mathrm{H}$ NMR $\left(600 \mathrm{MHz}, \mathrm{DMSO}-d_{6}\right) \delta 7.37$ (d, $J=10.8 \mathrm{~Hz}, 1 \mathrm{H}), 6.76(\mathrm{~d}, J=8.4 \mathrm{~Hz}, 1 \mathrm{H}), 6.56(\mathrm{~s}, 1 \mathrm{H}), 5.61(\mathrm{~s}, 2 \mathrm{H}), 3.59(\mathrm{~s}, 3 \mathrm{H})$. ESI-MS: 338.1(M+H) .

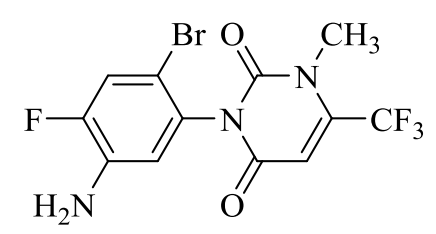

Data for 7c. Yield: $84 \%$, m.p. $170-171{ }^{\circ} \mathrm{C} .{ }^{1} \mathrm{H}$ NMR $\left(600 \mathrm{MHz}, \mathrm{DMSO}-d_{6}\right) \delta 7.33$ (d, $J=10.8 \mathrm{~Hz}, 1 \mathrm{H}), 6.68(\mathrm{~d}, J=8.4 \mathrm{~Hz}, 1 \mathrm{H}), 6.36(\mathrm{~s}, 1 \mathrm{H}), 5.56(\mathrm{~s}, 2 \mathrm{H}), 3.56(\mathrm{~s}, 3 \mathrm{H})$. ESI-MS: 382.0(M+H $)^{+}$. 
To a stirred solution of $7(0.02 \mathrm{~mol})$ in $50 \mathrm{~mL}$ of $\mathrm{DMF}$ was added potassium $O$-ethyl dithiocarbonate $(8.0 \mathrm{~g}, 0.05 \mathrm{~mol})$ at $90{ }^{\circ} \mathrm{C}$. The temperature was maintained at $90{ }^{\circ} \mathrm{C}$ for $6 \mathrm{~h}$, the reaction mixture was then poured into $500 \mathrm{~mL}$ of water and acidified by concentrated $\mathrm{HCl}$ solution to $\mathrm{pH} 3$ to induce precipitation. The precipitate was collected by filtration, washed with water and dried to obtain 3-(6'-substitued-2'-mercaptobenzo-[d]thiazol-5'-yl)-1-methyl-6-(trifluoromethyl)pyri midine-2,4-dione.<smiles>Cn1c(C(F)(F)F)cc(=O)n(-c2cc3nc(S)sc3cc2F)c1=O</smiles>

Data for 8a. Yield: $85 \%$, m.p. $114-116{ }^{\circ} \mathrm{C} .{ }^{1} \mathrm{H}$ NMR (600 MHz, DMSO- $\left.d_{6}\right) \delta 14.1(\mathrm{~s}$, 1H), $7.88(\mathrm{~d}, J=9.0 \mathrm{~Hz}, 1 \mathrm{H}), 7.43(\mathrm{~d}, J=6.0 \mathrm{~Hz}, 1 \mathrm{H}), 6.60(\mathrm{~s}, 1 \mathrm{H}), 3.42(\mathrm{~s}, 3 \mathrm{H})$. ESI-MS: 378.1(M+H $)^{+}$.<smiles>Cn1c(C(F)(F)F)cc(=O)n(-c2cc3nc(S)sc3cc2Cl)c1=O</smiles>

Data for 8b. Yield: 87\%, m.p. $124-125^{\circ} \mathrm{C} .{ }^{1} \mathrm{H}$ NMR (600 MHz, DMSO-d $) \delta 14.13$ (s, 1H), 8.09 (s, 1H), $7.48(\mathrm{~s}, 1 \mathrm{H}), 6.63(\mathrm{~s}, 1 \mathrm{H}), 3.45$ (s, 3H). ESI-MS: 394.1(M+H) .<smiles>Cn1c(C(F)(F)F)cc(=O)n(-c2cc3nc(S)sc3cc2Br)c1=O</smiles>

Data for 8c. Yield: 84\%, m.p. $133-135^{\circ} \mathrm{C} .{ }^{1} \mathrm{H}$ NMR (600 MHz, DMSO-d $) \delta 14.1$ (s, 1H), $8.20(\mathrm{~s}, 1 \mathrm{H}), 7.95(\mathrm{~s}, 1 \mathrm{H}), 6.63(\mathrm{~s}, 1 \mathrm{H}), 3.43(\mathrm{~s}, 3 \mathrm{H})$. ESI-MS: 438.0(M+H) 